\title{
Le « meitheal »
}

Un outil de formation

Peadar Cremin, Alain Dupré et Charly Ryan

\section{CpenEdition}

\section{Journals}

Édition électronique

URL : http://journals.openedition.org/ries/4047

DOI : 10.4000/ries.4047

ISSN : 2261-4265

Éditeur

Centre international d'études pédagogiques

Édition imprimée

Date de publication : 1 juin 1995

Pagination : 81-89

ISSN : 1254-4590

Référence électronique

Peadar Cremin, Alain Dupré et Charly Ryan, « Le « meitheal » », Revue internationale d'éducation de Sèvres [En ligne], 06 | 1995, mis en ligne le 01 janvier 2015, consulté le 03 mai 2019. URL : http:// journals.openedition.org/ries/4047 ; DOI : 10.4000/ries.4047

Ce document a été généré automatiquement le 3 mai 2019.

(c) Tous droits réservés 


\title{
Le « meitheal »
}

\author{
Un outil de formation
}

\section{Peadar Cremin, Alain Dupré et Charly Ryan}

1 À l'aube du III millénaire, après une longue histoire dont tous les pays européens portent les traces dans leur patrimoine, enfin l'Europe de l'éducation est en train de naître après celle des marchands, des politiques, des industriels. Cette forteresse Europe a besoin d'unir tous ses citoyens pour faire face aux défis du futur et cette perspective ne peut faire l'impasse sur la formation des personnes dès les premières années de scolarisation.

2 Déjà quelques initiatives d'associations d'enseignants avaient ouvert la voie de la coopération internationale dans le monde de l'éducation. Les Réseaux d'instituts de formation (RIF) veulent poursuivre cette action en lui donnant les formes les plus variées de la collaboration multiculturelle et en essayant de dépasser le cadre expérimental des premières actions pour modéliser et étendre le plus largement possible les expérimentations réussies.

3 Cet article, écrit par trois formateurs d'enseignants de trois pays différents (France, Irlande, Royaume-Uni) est un essai de mise au net après cinq années de réflexion et de travail, et au terme de trois expérimentations concrètes d'une formation initiale des enseignants intégrant la dimension européenne.

\section{Contexte de l'expérimentation}

\section{L'impulsion de la Communauté européenne}

C'est le 24 mai 1988 qu'une résolution sur la dimension européenne dans l'éducation a été adoptée. Il s'agit de « renforcer la dimension européenne dans l'éducation en lançant une série de mesures concertées pour la période 1988-1992 ", ainsi que de "renforcer chez les jeunes le sens de l'identité européenne et de leur faire comprendre la valeur de la civilisation européenne et des bases sur lesquelles les peuples européens entendent fonder aujourd'hui leur développement, à savoir notamment la sauvegarde des principes de la démocratie, de la justice sociale et du respect des droits de l'homme». 
5 Les deux applications aux États membres sont l'« intégration de la dimension européenne dans les systèmes éducatifs » et la " promotion de mesures visant à stimuler les contacts entre les élèves et les enseignants de pays différents ». Dans le paragraphe sur la formation des enseignants, il est prévu, au niveau de la Communauté européenne d'«utiliser les possibilités offertes par le programme Erasmus pour permettre aux étudiants suivant une formation d'enseignants de faire l'expérience d'un pays étranger dans le cadre de leur formation et pour intensifier la coopération entre les établissements de formation et les formateurs "; de "soutenir la coopération entre les établissements assurant une formation initiale et une formation continue aux enseignants de plusieurs États membres dans le cadre de la préparation du matériel didactique visant à promouvoir la dimension européenne dans l'enseignement»; de "promouvoir chaque année, pendant la période 1988-1992, l'organisation d'une université européenne d'été pour les formateurs, afin de leur permettre d'échanger leurs expériences et de définir de nouveaux moyens d'améliorer l'introduction de la dimension européenne dans la formation des enseignants ».

6 Au cours de l'Université européenne 1989 de Nimègue, sont nés les Réseaux d'instituts de formation (RIF). Ils se sont constitués sur la base de rencontres ainsi que sur l'intérêt manifesté par certains participants en regard de thèmes destinés à donner une direction de travail à l'introduction de la dimension européenne dans l'éducation.

\section{Les membres du réseau 5 , des meetings au « meitheal »}

7 Le réseau $n^{\circ} 5$ "Introduction de la dimension européenne à l'école primaire » s'est constitué à ce moment-là. Neuf institutions, mais en fait neuf personnes (une par institution) formaient l'équipe de base, coordonnée par Sister Angela Bugler du Mary Immaculate College de Limerick. Ce groupe compte actuellement seize institutions ${ }^{1}$.

\section{Démarche et stratégie}

8 La tâche du réseau était la préparation de futurs enseignants de l'école primaire à élaborer et à expérimenter un programme d'enseignement intégrant la dimension européenne. Alors qu'il est relativement facile d'apporter des idées nouvelles et d'imaginer du matériel pédagogique, l'histoire de l'éducation nous a appris qu'il était beaucoup plus difficile de changer les pratiques de classe.

9 Le point de vue des membres du réseau était que, d'une part, l'élaboration et la mise en œuvre d'un programme d'enseignement étaient aussi un processus et que, d'autre part, comme nous étions tous responsables d'un programme d'enseignement dans notre propre pratique, nous serions d'autant plus capables de faire le travail si nous constations que les activités étaient réalisables dans notre classe, avec des supports appropriés. Ceci serait encore plus probant si l'esprit de cette innovation était intégré à tous les outils d'enseignement. Ceci devait s'appliquer au matériel utilisé dans les écoles primaires et à celui des centres de formation. De plus, nous voulions vraiment prendre en compte les aspects soulignés par la résolution et relatifs à la coopération, la justice sociale et le respect des autres, avec toutes les implications que cela représentait. 
10 Au terme de nos réflexions, nous avons décidé de mettre en place une stratégie de type recherche-action à deux niveaux: celui de la formation des enseignants et celui de l'enseignement dans les écoles primaires. Ce compte rendu ne concerne que la formation des enseignants mais ses interactions avec d'autres niveaux seront aisément perçues: conséquences dans les classes auprès des élèves, effets auprès des formateurs du réseau eux-mêmes. Cette dernière conséquence fera l'objet d'une publication particulière du réseau $n^{\circ} 5$.

11 Ces considérations nous ont conduits à décider de réunir un groupe d'étudiants pendant un mois pour leur faire suivre un programme expérimental destiné à élaborer et expérimenter des séquences de classe introduisant la dimension européenne dans leur enseignement. Pour bien couvrir l'ensemble des disciplines du programme, des thèmes particuliers ont été déterminés pour chaque réunion d'étudiants : les arts, la géographie, les ressources du sol, etc.

12 Former les enseignants, c'est modifier leurs représentations. Dans notre situation, elles sont de quatre types: les connaissances concernant l'Europe, les valeurs de référence de chacun (principalement nationales), les idées sur l'enseignement et les savoirs de chacun pour chaque discipline, chaque sujet des contenus des programmes. Pour un apprentissage efficace, ces représentations doivent être identifiées, puis enrichies, transformées et évaluées. Cette démarche est facilitée par la mise en place de situations fortes, intenses, suffisamment longtemps (un mois), avec des objectifs clairs et ambitieux, un niveau d'exigence élevé qui puisse conduire à une action (faire la classe) dans un groupe multiculturel de pairs. Nous avons observé que cet aspect multiculturel a été déterminant, de même que la pratique avec des enfants.

\section{Objectifs}

Les formateurs avaient vécu un processus semblable pour concevoir les objectifs du mois pour les étudiants :

- améliorer les connaissances sur les systèmes éducatifs européens ;

- avoir une expérience concrète de travail dans une école primaire d'un État membre ;

- améliorer la compréhension de la nature et du rôle de la dimension européenne à l'école primaire ;

- établir des contacts personnels avec un certain nombre de futurs enseignants et avec des instituteurs expérimentés d'autres pays européens ;

- examiner ses propres valeurs et attitudes à travers une étude des points communs et des différences entre les différentes cultures représentées par les autres membres du groupe ;

- développer ses propres compétences professionnelles;

- encourager les liens entre les centres de formation d'enseignants et les écoles à travers toute l'Europe et imaginer une structure qui faciliterait de tels liens ;

- identifier des stratégies et des structures grâce auxquelles des thèmes et du matériel pédagogique concernant la dimension européenne à l'école primaire seraient conçus et diffusés.

La première session s'est déroulée à Winchester (1991), la seconde à Bourg-en-Bresse (1993), la troisième à Limerick (1994), la quatrième aura lieu à Copenhague (1995). Ces sessions ont rapidement pris le nom de meitheal. 


\section{Le « meitheal »}

L'origine du mot est celtique :

Traditionnellement, dans plusieurs pays européens, les paysans se réunissaient en automne pour préparer les moissons. Chacun à son tour sollicitait un groupe d'amis et de voisins qui venaient coopérer et l'aider à la tâche. Il n'était pas prévu de salaire, seulement le repas des ouvriers. Le service était récupéré à une autre occasion. Une tâche qui aurait été extrêmement lourde pour un seul devenait relativement aisée grâce au travail coopératif de plusieurs. Côte à côte dans l'effort, on observait une interaction sociale suivie d'une fête quand le travail était terminé. Cette tradition existe encore dans de nombreuses zones rurales et dans les pays celtes. En Irlande, elle est connue sous le nom de meitheal (prononciation irlandaise : $m e^{\prime}$ hal). ${ }^{2}$

16 C'est lors de la session de Winchester (1991) que Peadar Cremin a proposé ce mot aux étudiants du premier meitheal pour illustrer les caractéristiques de cette rencontre, car construire des démarches et du matériel d'enseignement dans une perspective d'éducation à la dimension européenne est plus facile et plus efficace dans un groupe qui travaille en coopération.

\section{Fonctionnement de l'expérimentation}

\section{Le contrat}

Il s'agit d'abord de la réunion d'un groupe d'étudiants et de formateurs pendant un mois au cours duquel un programme d'activités place les participants en situation de travailler ensemble dans un institut de formation d'enseignants et dans des écoles primaires voisines. Les étudiants ont préalablement effectué certains travaux : documentation sur l'Europe, connaissance de leur système éducatif, de la dimension européenne dans les programmes nationaux, préparations de leçons et d'exemples d'activités. Ils s'attendent à vivre quelque chose de particulier car, après avoir été sélectionnés à raison de deux par institut (les qualités de communication, d'ouverture d'esprit, une certaine aisance dans la langue du pays d'accueil étant nécessaires), ils sont informés par des documents préparatoires puis accueillis en internat collectif, dans le cadre d'un aménagement de la formation dans leur institut, s'intégrant à la formation habituelle du centre. Les étudiants ne paient que leur voyage aller-retour. L'hébergement collectif est un paramètre important car il assure la rencontre permanente entre les étudiants, la vie commune imposant des relations, des accords et permettant des projets de soirée, de week-end. Il convient donc de réunir les étudiants dans un logement collectif comportant des parties communes (cuisine, salle de travail ou de détente), des parties semi-collectives (les dortoirs) et des espaces individuels privés car ce type de résidence facilite les discussions et les débats entre les étudiants. Des moyens (salle de travail spécifique, équipements techniques, vidéo, reprographie, etc.) sont également mis à leur disposition.

18 Enfin, des enseignants, très ouverts à l'Europe, accueillent dans leurs classes, à titre expérimental, les futurs collègues venus pour prendre en charge des séquences de travail avec leurs élèves.

19 Les activités sont de trois ordres. Donner d'abord aux jeunes adultes des informations sur l'Europe, ses problématiques éducatives et leur faire vivre des confrontations de points de 
vue, des débats. Au cœur de ces débats se retrouvent les valeurs et idées de chacun concernant l'éducation en Europe et dans le monde. On peut alors soit les taire, soit les défendre. Préparer ensuite des séquences dans les classes qui sont réalisées en groupes d'étudiants de différents pays : préparation à l'institut, documentation, prise de contact avec les classes, séquences d'enseignement proprement dites, évaluations et analyses des prestations. Enfin, animer des périodes, plus ou moins libres, qui se déroulent en dehors du programme pédagogique : soirées, week-ends, après-midi libérés, échanges entre les sessions de travail. Quelques propositions sont faites par les tuteurs (découverte de la ville, des environs, patrimoine culturel du pays), mais les étudiants ont largement la possibilité de construire des projets.

Par ailleurs, des documents d'accompagnement préparent les étudiants à leur séjour, sous forme de " passeports ", fascicules de présentation, destinés à favoriser une connaissance préalable des participants. Un programme détaillé avec les activités, leurs objectifs et l'emploi du temps heure par heure leur est également fourni. Cet agenda qui a permis aux étudiants d'avoir une première idée de ce qu'allait être leur séjour est négocié chaque semaine pour apporter les modifications rendues nécessaires par le déroulement du travail.

\section{Modalités d'évaluation}

21 Les questionnaires individuels sont distribués, remplis et commentés à l'issue de chaque semaine et à la fin du meitheal. Ils se réfèrent explicitement aux objectifs (atteints ou non) assignés pour chaque partie du programme. Une étude statistique des réponses est faite systématiquement par les tuteurs.

22 Les productions pédagogiques sont réalisées sous forme de fiches rédigées à partir de séances en classe. Elles s'organisent en trois ou quatre séquences d'enseignement effectuées sur plusieurs jours, comportant une synthèse la dernière semaine. L'objectif du réseau est de construire un document regroupant toutes ces productions selon le niveau de classe et comportant toutes les informations nécessaires à un enseignant pour les mettre en œuvre : niveau de classe, objectifs, consignes de travail, matériel nécessaire, déroulement, évaluation, prolongements, etc. Les différents supports des travaux de classe (cartes, affiches, vidéos, livres) sont également collectés. Les journaux professionnels sont utilisés par les étudiants pour s'exprimer par écrit (des plages de temps sont aménagées à cet effet) et formuler leurs opinions, impressions, réactions, afin, s'ils le souhaitent, de communiquer ces écrits aux tuteurs et dialoguer avec eux.

23 À l'issue du séjour, un document tenant lieu de certification est remis à chaque participant pour attester de son assiduité, de son engagement dans les activités, de la valeur du travail qu'il a réalisé. Actuellement, il a surtout une valeur symbolique.

24 Enfin, les maîtres des classes d'accueil contribuent à ces évaluations, en notant l'élan impulsé auprès des enfants par la venue des étudiants dans leurs classes. Cet élan connait des prolongements après le départ des étudiants, tant auprès des maîtres eux-mêmes que des parents d'élèves.

\section{Les apports en termes de formation}

25 Les relations privilégiées avec les autres étudiants, le vécu intense, le fait d'avoir ensemble relevé différents défis et participé à de nombreux événements inhabituels, 
l'enrichissement d'avoir côtoyé durant un mois une vingtaine de personnalités de cultures différentes, échangé, enduré ensemble les mêmes inquiétudes, pu (ou osé) présenter son pays, défendu ses idées, en un mot, vécu un peu hors du temps une aventure unique, tout ceci a transformé les personnes d'une manière forte et durable.

De plus, pour ce qui concerne directement le travail de l'enseignant, la richesse de l'expérience, la découverte d'autres systèmes éducatifs et d'autres façons de penser, le travail dans plusieurs langues différentes, l'échange sur les représentations du métier d'enseignant, la conduite de séquences de classe, la remise en cause de son style pédagogique conduisent à relativiser des certitudes professionnelles, par exemple: l'évaluation, la préparation, la démarche en classe, la relation aux élèves. Il développe en outre une meilleure réflexion sur l'acte d'enseignement: la méthode idéale n'existe pas; la confrontation est une situation de progrès individuel ; le travail de groupe est un levier du véritable apprentissage.

Plusieurs facteurs peuvent expliquer ces aspects positifs: des conditions de travail satisfaisantes, lieux de vie et de travail adaptés, matériel à proximité, encadrement présent; des conditions de vie rassurantes, structurées, propices à l'échange, ouvertes aux initiatives; des relations humaines appréciées, facilitation et accueil des personnes rencontrées, équipe de formateurs soudée.

Chaque institution est représentée pendant le meitheal par un formateur qui participe à l'encadrement pendant une durée de deux semaines (ou quatre semaines pour celui de l'institution d'accueil).

Un partage du travail a été effectué avant le meitheal et chaque formateur a donc une mission assez précise à assurer dans le déroulement des activités : exposé sur un sujet particulier, conduite du groupe, gestion des évaluations, des journaux professionnels, aide aux étudiants pour préparer les séquences de classe ou pour avoir un échange personnel.

30 Les réunions de formateurs ont permis d'échanger des points de vue sur les questions posées aux étudiants pendant le meitheal (en particulier: qu'est-ce que la dimension européenne?); de préparer un agenda précis et détaillé, de préciser le profil des étudiants et de construire un fascicule de présentation (les " passeports »); de répartir les différentes tâches de chacun; de travailler à bien préciser la nature et l'utilisation du journal professionnel, car ce document est une source d'informations très précieuse pour réguler la vie du groupe ainsi que pour les évaluations finales et la rédaction du rapport général par les formateurs.

31 Toutefois, des difficultés professionnelles et personnelles réelles doivent être relevées concernant la langue, la préparation de la classe, la communication, la découverte d'une culture, le débat d'idées pouvant aboutir à un conflit. Une atmosphère particulière, faite à la fois d'une familiarité progressive, mais également d'une pression - contraintes horaires, obligation de conduire des activités de classe, de coopérer - et surtout d'une énigme: qu'est-ce donc que la dimension européenne? Est-ce un ensemble de connaissances, un contenu ou n'est-ce pas plutôt un processus, une histoire qu'il faut vivre pour en mesurer la dimension dans toutes ses composantes?

Cet ensemble complexe contribue à développer un sentiment ambivalent : le plaisir d'être là, la difficulté de supporter le groupe pendant un mois et l'irritation de maîtriser si difficilement le concept ainsi que, simultanément, un sentiment de dépassement de soi 
(quand tous les objectifs sont atteints) conduisent à vivre le séjour comme une conjonction d'émotions contradictoires.

\section{Bilan global}

Les coûts, en personnes, en temps et en écus, la non-reconnaissance dans le pays d'origine, l'absence de suivi officiel, quelques difficultés de fonctionnement, peuvent être inscrits au passif de l'opération. Toutefois, les aspects positifs de l'opération doivent être soulignés et concernent les modifications des futurs enseignants, la production de matériel pédagogique, la force et la solidarité des formateurs.

Si l'on veut garder ce qui fait la force du meitheal en adaptant ses atouts à un réalisme qui impose moins de moyens et moins de temps, on peut imaginer certaines formules.

- Le mini-meitheal : il convient alors de conserver cinq principes : le groupe multiculturel, le séjour dans le pays étranger, une tâche à réaliser, une exigence de résultat, un encadrement vigilant et aidant. Un séminaire de deux semaines, ou trois, en utilisant une partie d'une période de congé, est tout à fait envisageable. Pour limiter les frais d'hébergement, l'accueil chez des étudiants (ce qui impose de trouver autant d'étudiants du pays d'accueil que d'étudiants étrangers) ou une prise en charge financière à $50 \%$ (en logements universitaires) devraient être possibles.

- Le travail coopératif: des groupes de quelques étudiants de pays différents pourraient correspondre régulièrement pour élaborer des situations de travail dans les classes de leur pays, grâce à des documents ou du matériel envoyés mutuellement. Ces situations seraient expérimentées et une évaluation adressée aux correspondants. Un formateur assurerait, dans chaque centre, la bonne marche du dispositif. La télécopie serait là un outil intéressant pour travailler en temps réel et échapper à la lenteur démobilisatrice de la correspondance postale. La vidéo, la photo, les enregistrements sonores, les dossiers, les produits de la vie courante sont autant de supports qui enrichiraient ces échanges. Une rencontre finale brève entre les jeunes étudiants permettrait un échange de vues et un bilan du travail effectué.

En réalisant ces trois expérimentations, nous avons peu à peu amélioré le déroulement : alternance des temps de travail et des temps libres, dialogue avec les étudiants à l'aide du journal professionnel, gestion des séances de travail en groupe, utilisation de la vidéo ${ }^{3}$, précision des consignes pour les activités. Mais nous avons aussi progressivement mesuré la force du dispositif, son impact auprès des jeunes. La constitution d'un microcosme européen avec sa richesse et sa diversité, comme ses risques de différends et de conflits, est un temps important et essentiel d'un processus d'analyse et de recherche-action pour répondre à la tâche du réseau: mettre au point des stratégies et des outils pour développer l'éducation à la dimension européenne à l'école primaire et dans la formation initiale et continue. 


\section{ANNEXES}

Liste des institutions du réseau $\mathrm{n}^{\circ} 5$

1. Allemagne, Oldenburg Universität, Oldenburg

2. Belgique, Institut d'enseignement supérieur, Namur

3. Danemark, KDAS, Copenhague

4. Espagne, Escuela Universitaria, Madrid

5. France, IUFM de Lyon, Bourg-en-Bresse

6. France, IUFM de Paris-Les-Batignolles, Paris

7. Grèce, Patras University, Patras

8. Irlande, Mary Immaculate College, Limerick

9. Pays-Bas, Hogeschool Gelderland, Nimègue

10. Pays-Bas, Pabo Thomas More, Rotterdam

11. Portugal, Escola Superior de Educaçao, Braga

12. Royaume-Uni, King's Alfred College, Winchester

13. Royaume-Uni, Worcester College, Worcester

14. Royaume-Uni, Nottingham Trent University, Nottingham

15. Royaume-Uni, Stranmillis College, Belfast

16. Royaume-Uni, Northern College, Aberdeen

\section{NOTES}

1. Voir cette liste en fin d'article.

2. Texte de Peadar Cremin.

3. Celle réalisée à Bourg-en-Bresse est disponible auprès de A. Dupré.

\section{RÉSUMÉS}

Les auteurs analysent des expériences de formation initiale d'enseignants du primaire : le rôle déterminant du groupe multiculturel de pairs et des modules intensifs axés sur un projet d'élaboration et d'expérimentation de matériel pédagogique; les apports et les limites - coût, absence de validation par les institutions d'origine. 
INDEX

Index géographique : Europe

Mots-clés : dimension européenne, enseignement primaire, formation des enseignants, pluralisme culturel, outil pédagogique

\section{AUTEURS}

\section{PEADAR CREMIN}

Head of the Education Department, Mary Immaculate College, Limerick, Irlande.

\section{ALAIN DUPRÉ}

Inspecteur-Formateur, IUFM de Lyon, Centre de Bourg-en-Bresse, France.

\section{CHARLY RYAN}

Senior Lecturer in Professional Studies, King Alfred's College, Science Department, Winchester, Grande-Bretagne. 